\section{A Cliniral}

\section{THE DETECTION OF EARLY TUBERCLE IN THE LUNGS.}

Delivered at St. George's Hospital.

By Sir ISAMBARD OWEN, M.A., M.D., F.R.C.P., Physiclan to, and Lecturer on the Principles and Practice of Medicine at, the Hospital.

In the year 1901, the last for which the Registrar-General's returns are available, there died in Englund and Wales 316,997 persons over the age of 20 , of whom 35,526 , or about one in every nine, were stated to owe their death to "phthisis" or "pulmonary tuberculosis." And these deaths are not deaths of old people, whose course was in any case nearly run. The victim of pulmonary tubercle is nearly always cut off in what should have been the most active period of his life, and usually after years of more or less complete disablement. Of this mass of suffering and premature death, repeating itself as it does year after year, much, perhaps the greater part, may be regarded as preventable, if proper treatment of the case be resorted to in time. But the condition of time is all important. Every month during which active tuberculous disease remains undetected and untreated lessens appreciably the prospect of recovery. I think, therefore, I am not exaggerating in saying that nothing in the art of medicine is of greater importance to acquire than the power of detecting with precision the early presence of this dangerous growth in the lung. For the means of doing so you have still chiefly to rely on the now old-fashioned means of physical examination. Bacteriology has supplemented, but cannot replace them. Even on the bare question of the presence or absence of tubercle in a lung it often fails us; on questions of the age, extent, distribution and histological character of the tuberculous lesions it can tell us little. The Roentgen ray is the latest addition to our implements of exploration. Though it is at present in its infancy it bids fair to render us valuable assistance; but, so far at least, we have little reason to think that it will ever rival the fingers and the ears for delicacy and exactitude of diagnosis.

The tuberculous lesions you have to look for are often of small size; they are not always on the surface of the lung; the signs they afford frequently involve such fine distinctions of sounds as only a well-trained ear can appreciate. You have not only to detect their presence when they exist; you need also, which is the more difficult, to make sure of their absence when they do not. Such training of the ear as is required can only be gained by constant practice; practice not in the wards alone, but in the ampler field of experience furnished by the receiving-rooms and out-patient department. Even that will avail you little unless you pursue your study of the subject in the post-mortem room as well, and familiarize yourselves with the actual histological conditions, manifold and various as they are, of which the physical sigus you discover should afford you a mental picture. Post-mortem observation will in the first place guard you from the common, but incorrect and misleading, use of the word "stage" in reference to cases of pulmonary tubercle. One case, we sometimes hear it gaid, is " in the stage of grey granulation," another "in the stage of caseation," a third "in the stage of breaking down" or "in the stage of cavitation." The specimens of tuberculous lungs which I am about to lay before you will, I think, show you the inadequacy of such a terminology. The morbid process is seldom one that advances along a continuous and uniform course in all parts of the lung affected, even though the area of affection be very limited. The morbid histology of nearly all these specimens is, as you will see, a mixed one; and few can be said, even predominantly, to represent any single "stage" of the process. The word " stage," in fact, belongs to the local, not to the general; histology of the disease. I will ask you then to dismiss the'idea of definite clinical "stages" of pulmonary phthisis from your minds, and will in its stead invite your reception of 'annther idea which may possibly appear more novel, as it is seldom adequately brought out in the literature - of tuberculosis. I mean the idea, or the fact, as I will claim to call it, that pulmonary tuberculosis in most of its ordinary forms is a disease of essentially intermittent or remittent character. That : it is not so in all cases I admit. In a minority of instances it runs a steadily progressive course from onset to fatal termination; but in the majority, if only you have the opportunity of watching them from the beginning, you will find at first a frank intermittence of the active process, and to a very late period a spontaneous tendency to fluctuations or remissions of its severity.

Whether the materies morbi obtains admission to the lung by the air passages, as is generally held, or, as some pathologists bekeve, by the lymphatic channels, the primary attack of tubercle upon the lungs is, in most cases, at first a narrowly localized one, and the infected area does not extend with any great rapidity. The bacillus seldom enters the blood stream ; the disease in its earliest period advances by the propagation of successive broods or by the slow passage of the germs in the adjacent lymph spaces; extension even by the air passages is a phenomenon belonging rather to a later period of the case. The tubercle bacillus, if we may judge by clinical observation, is not exempt from the rule so commonly applying to pathogenic germs, that in attacking the human tissues they breed the means of their own destruction; so that the individual attack of tubercle, if it be of limited extent, comes spontaneously to an end after a given space of time, just as an attack of scarlatina or measles would. Upon the destruction of the materies morbi and the consequen cessation of the active morbid process, the granulation tissue which is the main element of the grey nodules, supposing it to be only grey nodules that the disease has yet produced, follows the usual course of such tissue and becomes converted into fibrous scar. Puckered scars, now fully recognized as the handiwork of tuberculosis, though they contain no trace of the specific germ, are frequently found in the apices of the lungs. of persons who have never been known to have suffered from pulmonary disease. In such cases, it is now generally admitted, a tuberculous process must have taken place at some unknown time of life, possibly in early childhood, though the germ has entirely disappeared and the attack has resulted in nothing but a harmless and noninfectious scar. This spontaneous arrest of the disease in its early period is by no means exceptional. Taking necropsies of bodies which presented no other evidence of tubercle, the proportion in which the scars of healed tubercle are found is stated by some authors at even so high a figure as 30 per cent. If we take it at only a third of this figure, reoovery from early pulmonary tubercle would seem to be as frequent a result as death.

When tubercle attacks a serous membrane, and the patient survives the immediate attack, the tendency of the new growth towards permanent arrest is evident. Tubercle of the pleura is far from being a rare form of disease. In the majority of cases the patient recovers with a pleura firmly adherent and generally thickened. The subject of tuberculous peritonitis also frequently recovers with an adherent peritoneum. Neither in these cases, nor in the rarer cases of survival from meningeal tubercle, is there often seen a relapse or a recurrence of the malady.

In the loose tissue of the lung, on the other hand, tubercle, even of the firm grey variety, tends to aggregate into larger nodules which coalesce to form continuous areas of consolidation prone to degenerate and become caseous at their centres. The products of tuberculous broncho-pneumonia, a form of tuberculous process seldom altogether absent from a case of the disease, caseate with even greater readiness. Caseous matter is unorganizable; in large masses it is unabsorbable; and when an accumulation of a certain size has been formed complete cicatrization is no longer possible. Fibrous organization, if it occurs, takes place only at the uncaseated periphery of the mass, and the caseous material, or some part of it, remains permanently embedded in a tract or capsule of fibrous tissue and suspended thereby in the midst of the loose pulmonary web. Now caseous material (it is one of the earliest experimental observations on the disease) remains permanently infectious. Though the tubercle bacillus can rarely be detected in it by a microscope it can be readily culttvated from it in the body of a susceptible animal. In the human lung, hemmed in by its cicatricial capsule, caseous matter may remain for a longer or shorter period without harming its surroundings ; but, unless by good fortune it should becupice calcified, it retains its power of reinfection and sooner o "later, under circumstances not at present traceable it does, in the majority of cases, reinfect the

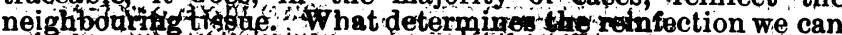
only guegs 61 in 3 it often appons connected with a

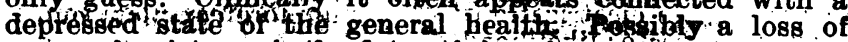
power of resistance is the determining circumstance; possibly some failure of nutrition of the fibrous tisiue, by relaxing the

[2257] 
ure the capsule normally exerts on the caseous mass,

ows it to absorb the degrce of moisture necessary for the active life of the germ. In serous membrines, no liss than in the lung, tuberculous tissue mag caseate; it is the common tendency of tubercle wherever it occurs: but the individual caseous masses are smaller in size and, even if encapsuled instead of being absorbed, are left embedded in firmer fibrous tissue than is the case with the caseous matter remaining in the lung.

The fresh tuberculous tisgue formed in the lung by reinfection, and even that formed in several successive reinfections, may follow (I am again inferring from clinical observations), the same course of growth, caseation, arrest and peripheral cicatrization as the original lesions. Between each successive attack, as long as the lesions renain of small extent, the symptoms may entirely subside and definite intermissions be established. In these intermissions the bacilli may entirely disappear from the fauces; in some cases they are still to be detected in small numbers. If the attacks continue, a time comes when the tuberculous formations have reached such an extent that their progress is no longer uniformly synchronous; active processes are always present in one part or another of them, and the clinical course of the case becomes remittent rather than intermittent. In many cases the remittent character is more or less traceable almost to the end, and in not a few cases complete intermissions, as far at least as symptonis are concerned, appear at a surprisingly advanced period of the case.

Large as is the proportion of cases in which the intermittent onset of tubercle, as I have described it, is actually observed, it is a fair clinical inference that it would be much larger had we more often the opportunity of watching tuberculosis of the lung from the beginning. Except in cases where early haemoptysis draws attention to the lungs it is doubtful if we often really see the initial attack at all. A slight attack of tubercle may cause either no symptoms or none that will lead the patient to seek medical advice. As I have already said, scars of healed tubercle are found in the lungs of persons who were never known to have suffered from pulmonary disease. Early haemoptysis, when it occurs, often reveals the real nature of an illness that would otherwise have passed for a temporary indisposition. In some cases, indeed, there has been no perceptible symptom whatever before the haemorrhage appeared. Such cases, thanks to the haemorrhage, are brought, and generally kept, under medical observation. Failing the blood spitting they would have escaped professional notice till long after the real onset. perhaps not till one, two, or more periods of intermission had passed by. Let me instance a patient at present under my care in the William King IVard, who presents a small area of consolidation, apparently quiescent, in the right apex, evidenced by harsh breathing and comparative dullness in the subclavian fossa and first intercostal space. He has had, he tells us, three slight attacks of haemoptysis, if not four, in the course of the last four years; each of them, he says, was accompanied or followed by a slight cough; but the cough never lasted more than two or three weeks, and his general health was so little affected that he served in the army through the greater part of the South African war. Except in the first attack of haemoptysis he never laid up or sought medical advice on his case. But though it is largely, it is by no means exclusively these haemorrhagic cases in which the frankly intermittent onset is actually seen. If the lungs of roung people should ever become the object of as much solicitude as their teeth, and be habitually subjected, like them, to periodic precautionary examination, the detection of tubercle in its earliest period will doubtless be much more frequent than it is at present, and the prevalence of intermittent onset be more generally recognized.

The essential intermission and remission of pulmonary tubercle would probably, indeed, have attracted more attention already than has actually been the case were it not for our inveterate tendency to attribute any improvement that may occur in a case of serious disease to the effects of treatment. Pray do not imagine that I wish to decry the importance of treatment in tuberculous disease. Were treatment of no avail it would be waste of labour to speak of early diagnosis. Judicious management, there is reason to think, may both limit the extension of tubercle during the attack and shorten its duration. What is more certain and more to the point is that good treatment may prolong the period of intermission 80 as to enable cicatrization to become more complete and to acquire a greater likelihood of permanency; but intermission and remiseion, I am anxious to impress upon you, are among the natural features of the disease; they may be observed even in cases placed under unlavourable circumstances. in cases subjected tu almost any reasonable treatment, and in persons who have received, like my soldier vatient, no particular treatment at all.

The result of this is that in most case's of chronic tubercle of the lungs that actually come under the physician's hands even for the first time we have already a mixed morbid anatomy present; we may have some fibrous scar tissue and we may have some caseous material, and both of these may include portions of different date. If the case is seen during the period of activity, we probably have also some recent grey granulations, with which more or less broncho-pneumonia is likely to be sssociated. If there is much fibrous scar there will presumably be some compensatory emphysema. In addition. though rarely in such early cases as those we are speaking of there may be one or more cavities, and if the tubercle, as often happens, has been partly superficial, the pleura will be in places adherent and greatly thickened. There are, of course, exceptions. In very early cases grey nodules alone may be present. In some cases, particularly in children, the entire lesion is broncho-pncumonic from the first, and caseates rapidly without producing either grey granulation or fibrous scar. Broncho-pneumonic cases, let me say in passing, are likely to be exceptions to the rule of intermission and remission, and to run a continuous course from the first.

The whole of the above-named lesions, cavities and emphygema apart, may for the initial purposes of physical diagnosis be summed up in the single word "consolidation." Chronic partial consolfdation of the apical portions of the lungs is seldom anjthing but tuberculous, and the preliminary search for signs of tubercle is, to a large extent, a search for signs of consolidation. According to the time-honoured tradition of physical examination the search should commence with inspection, should proceed to palpation, then to percussion and last of all to auscultation. May I be so unconventiona) as to say that the traditional order of procedure savours far more of pedantry than of practical convenience? There are cases in which the signs furnished by the fingers are clearer and more definite than tlose obtained by the stethoscope; but there are also cases in which the reverse obtains, and the latter class of cases far outnumbers the former. On the whole, therefore, a considerable economy of time is effected by the method I usually parsue of commencing, after a cursory inspection, with a thorough stethoscopic exploration of the upper parts of the lung, and following it up with percussion and with a more detailed inspection and palpation. I have more than once, in a clinical examination, seen a candidate spend nearly the whole of his allotted time in attempting to settle the relative dullness of two apices, the tuberculous condition of both of which was obvious the moment the stethoscope was applied. Prolonged percussion, too, is liable to cause a certain amount of suffering to the patient, which is best avoided when not really necessary. The direct signs of consolidation. I need hardly remind you, are the presence of what is called " tubular," "bronchial," or " harsh "breathing, of changes in the voice sounds which may vary from mere ezaggeration to nasal " bronchophony," and of dullness on percussion as compared with the opposite side. In their typical form these signs are the A.B.C. of physical examination, but in the search for tubercle we find them in 80 many different degrees, combinations, and doubtful forms that I will ask your attention to a few preliminary considerations before discussing the detailed interpretation of them.

Tubalar breathing (I prefer "tubular" to "bronchial," as it involves no theory) is a sound which may be fairly imitated by blowing or, rather, by drawing air in and blowing it out, through a narrow straight pipe, such as the tube of a wooden stethoscope. It may be more nearly simulated by breathing through the mouth while the back of the tongue is raised towards the soft palate, so as to reduce the faucial aperture to a narrow horizontal slit; and by slight changes in the position of the tongue the simulated sound may be made to follow all the variations of pitch which tubular breathing assumes under different circumstances. It is generally recognized that the actual sound of tubular breathing is produced, not in the chest, but in the larynx or its near neighbourhood, and that it is conducted downwards by the column of air in the traches and bronchial tubes. Generally speaking, it is not heard over the surface of the healthy chest, being intercepted by the surface stratum of pulmonary tissue, which contains no tubes. In some persons, even in health, it may be more or less discerned in the interscapalar region or over the main bronchi in front but it is nat correct to say, as some anthors do, that it is 
identical with the sound heard when the stethoscope is placed over the trachea; there is an important difference, to which I shall allude later. The sound, then, as I have said, is conducted by way of the trachea and main bronchus to the root of the lung, and thence along the lesser bronchi to within a certain distance of the surface, but in the healthy lung does not reach the surface. The hearing of well-marked tubular breathing over the chest wall implies that over the area covered by the stethoscope the superficial layer, at least, of the lung is sufficiently consolidated to conduct the sound to the surface from the point it normally reaches in the tubes and at the same time to abolish the natural vesicular murmur. Very superficial consolidation which does not reach as far as the deeper layer of the lung cannot carry forward the tubular sound. Consolidation of the deeper part of the superficial layer, not extending to the surface, may carry the vibrations forward so far as to convey a faint or "distant" tubular sound; a chain of consolidated points, leaving some permeable lung in the area covered by the stethoscope, brings a sort of diluted tubular breathing to the ear, a mixture of a faint tubular breathing with the normal vesicular murmur, which is generally called "harsh" breathing. Though other causes than consolidation in rare instances convey a tubular or harsh character to the breath sounds, the distinetion between true and false tubular breathing is easy, if the expiratory part be taken as the criterion. If the expiratory sound, having the tubular or harsh character, is found to proceed with uniform pitch and intensity through the whole expiratory act, the breathing is truly "tubular" and is evidence of consolidation. If it does not satisfy this criterion, if it varies in tone, or "tails off" towards the end, it only indicates some trifling bronchial change. Mere prolongation of the expiratory sound, without the tubular or harsh character being manifest, does not constitute evidence of consolidation at all; the expiratory sound is equally prolonged in some forms of false tubular breathing, in puerile breathing, and in the clear "blowing breathing" which is so often met with over healthy or merely emphysematous apices, and which is probably of bronchial origin also. These sounds must not be confounded with the true consolidation signs, and the use of the vague term "prolonged expiration" in describing physical signs is, therefore, in my opinion, not to be recommended.

I will ask you now to note that the pitch of the harsh or tubular breathing heard in early tubercle of the apex is nearly always low-much lower than is commonly heard in pneumonic consolidation of the al ex or over lung compressed by pleural effusion. The pitch of tubular breathing is a subject to which little attention has been given, but you will find it a point of some practical value. Several considerations, which it would be too long to enter on here, have led me to believe that high-pitched tubnlar breathing is only produced when there is a continuous tract of consolidation between the point at which the stethoscope or ear is applied and the neighbourhood of the root of the lung. This seldom happens in early tubercle; the existence of high-pitched tubular breathing, if anywhere found, would, on this view, be of serious import as regards the extent of the lesion. In speaking of the "pitch" of tubular breathing, you will, of course, understand that, neither as produced in the throat nor as heard over the lung, is tubular breathing ever a definite musical note. It is always a mere "noise," compounded of vibrations of indefinitely various lengths, some of which are better conducted under some circumstances and others under other eircumstances. In speaking of tubular breathing "pitch" must be taken to mean the average pitch of those elements of the sound that most obviously impress themselves on the ear.

What the actual depth may be of the layer of the lung that I am describing as "superficial" is difficult to say. There is reason to think that it does not exceed half an inch. Con solidation entirely below this depth, whatever the depth may really be, will bring no tubular or harsh breathing to the ear. It does not therefore follow that the breath sounds give us no information at all as to the existence of deeper consolidations. If consolidation exists in large amount it.hinder either the expansion of the apex of the lung or the access of air to its more superficial parts, and its presenoe at a deepe level is aecordingly often evidenced by diminution of the breath sounds or bv an irregularity of the inspiratory rhythm ("wavy breathing"), even though the actual character of the sounds may be quite normal. You need, therefore, to com pare the breath sounds on the two sides for intensity and rhythm as well as for character.

The consolidation signs derived from the voice sounds are not always uniformly coupled with those derived from the breath sounds. Bronchophony is a natural sound produced, like tubular breathing, in the larynx, modified into articulate speech by the upper air passages, and carried by conduction along the column of bronchial air:; but the superficial layer of lung does not cut it off so entirely as it cuts off the tubular breath sound. The vocal sounds, as might be expected from their relative loudness, are more readily transmitted than the respiratory murmurs, and instead of being stopped by the superficial layer are merely transformed into the inarticulate "buzz" that we know as "vocal resonance." Deeply-seated consolidation may augment the transmission of voice sounds without preventing their normal transformation, and thus we get "increased vocal resonance." Increased loudness of the normal vocal resonance may therefore result from consolidations which are too deeply seated to give even "distant" tubular breathing; and we may obtain from it an indication of a deep consolidation even when the breath sounds fail us. On the other hand, we so frequently come upon patches of harsh breathing which are unassociated with any change in the voice sound. that we must conclude a more extensive or more complete consolidation to be needed to bring bronchophony to the surface than suffices to transmit the tubular breath sound. You will therefore regard harsh breathing associated with voice changes as of more serious import than harsh breath ing without such changes, and understand that well-marked bronchophony must indicate a rather extensive tract of consolidation. Genuine bronchophony without associated change in the breath sounds is very rarely met with. I need hardly remind you of the allowance to be made for the normal excess in vocal resonance of the right chest as compared with the left. As this is an uncertain quantity, great caution must be used in drawing conclusions from an apparent increase of voice sound at the right apex, unless its character be changed or other signs concur. Vocal fremitus is also normally more marked, often greatly more marked, at the right apex than at the left, but the value of vocal fremitus in the diagnosis of early lesions is practically nil, and it need not detain us.

Percussion for the detection of tuberculous consolidation requires to be practised with great care and its results to be interpreted .with judgement. To percuss the apices satisfactorily, it is desirable to have the patient standing before one. In no other posture is it equally possible to plant the plessimeter finger in a corresponding position on the two sides. But in merely placing the plessimeter finger in a similar position on the two sides there lies a snare, for, if the shape of the two sides is not quite alike, the result is vitiated. See therefore that you are percussing over corresponding points on the two sides, that you are using, as far as you can judge, the game percussive force, and that you are percussing upon exactly the same spot of the plessimeter finger, but otherwise vary the precise attitude and the degree of pressure of the latter until you are sure that you have elicited at each spot the best note that is to be got under the conditions, and compare the best notes thus obtained.

The results of percussion in many cases appear to correspond but ill with those of auscaltation. In some cases we find little or no loss of resonance where auscultatory signs of consolidation are well marked, and in others marked dullness where little or no change in the breath or voice sounds has been found. In the former of these cases the presumption is usually that the resonance of the affected part is due to compensatory emphysema, which argues that the consolidation consists largely of fibrous scar. In some such instances the affected, or the more affected, apex is actually the more resonant of the two. In the latter case there are several hypotheses to be considered. "Deep" percussion gives indications of consolidation at a level too deep for it to furnish atethoscopic signs, either respiratory or vocal. In the first place, therefore, there way be consolidation deeply situated. Secondly, the presence of abundant scattered grey tubercle may affect the percussion resonance of the lung without making the breath sound harsh or increasing the conduction of the voice, these being effects which need continuous consolidation to produce them; and, thirdly, the pleara at the spot may be greatly thickened.' Now comes in the value of systematically practising both light and deep percussion and comparing the results. . If the dullness is more marked with the light than with the deep percussion, it is probably a thickened pleura that causes it; if the reverse obtains, it suggests a rather deeply-seated patch of consolidation. In the case of scattered grey tubercle the results of the two kinds of percussion will vary little.

To return to the subject of compensators emphysema in the 
neighbourhood of fibrous scars ; you may be puzzled now and again by the curious changes in the physical signs that sometimes take place from day to day or from week to week in apices that are the seat of quiescent tubercle. One day you may find a distinct patch of dullness, associated with clearly defined harsh breathing and possibly some increase of vocal resonance. The next time you listen, neither dullness nor harsh breathing are to be found. Or, which is more embarrassing, the change may be in the reverse direction without any symptom whatever to suggest the onset of fresh tuberculous disease. The most plausible explanation of this seems to be that the change is due to variations in the degree to which the emphysematous lobules are at the moment distended with air. On one day more active respiratory movements may have caused them to become fully distended, so that the conductivity of the lung for sound is lessened and the percussion note improved. On another day partial collapse may reduce the percussion resonance and allow consolidation sounds to penetrate. The explanation is merely hypothetical, but the occasional occurrence of these changes is a fact you will do well to remember.

To determine the existence and superficial extent of a limited tuberculous consolidation is something; but.it is equally important to know what it really means. How far does it consist of grey granulations; of ffibrous scar; of broncho-pneumonic products? Is it wholly quiescent, or anywhere actively advancing? How far can physical examination help us to answer these questions? The advance of tubercle at a given spot may take place by the formation of outlying grey granulations or by the extension of a broncho-pneumonic area. In the former case, provided that the granulations are sufficiently near the surface, the determination of an active condition is comparatively easy ; for each active granulation is surrounded by a zone of congestion with some localized fluid exudation into the alveoli or small bronchi that generally gives rise to "moist" sounds during the inspiratory expansion of the lung. Do not fall into the common error of supposing that moist sounds in a tuberculous lung necessarily indicate "breaking down." The smaller moist sounds do not in the least necessarily do so; they equally accompany the very earliest stage of grey tuberculous nodule. They may be equally found where only scattered granulations exist and where older lesions are extending by grey granulations at their edges. Two kinds of small moist sound may attend advancing grey granulations, the crepitation that shows the localized exudation to be merely serous or oedematous, and the sharp, sticky click that bespeaks it inflammatory or bronchopneumonic. Crepitations need to be distinguished from the friction sound of tuberculous pleurisy-a matter of small importance-and from the dry, dull crackle of apical emphysema ; a distinction of serious consequence, but one which practiole enables one easily to make. Clicks at the end of inspiration are formally diagnostic of tubercle; but they may occur at the commencement of "breaking down" as well as around active granulations. The interpretation will depend on their situation. If they are heard over a patch of detinite consolidation, they are probably due to disintegration; if at the edges of such a patch or at a distance from it, they indicate an early stage of an advancing lesion. Both the crepitations and the clicks are high pitched, and neither, as may easily be demonstrated, are conducted far from their seat of origin. Granulations more than a short distance below the surface, it may therefore be inferred, do not reveal themselves in this manner. "Large" râles which possess a low pitch, such as the larger mucous râles of general bronchitis, are, on the contrary, well conducted through lung tissue, and may be heard on the surface, though actually formed in the larger bronchi.

Tuberculous crepitations may be accompanied by light, dry wheezing rhonchi. In some cases the rhonchi may be heard even though crepitations are not audible. The conductivity of the lung for rhonchus is much greater than for crepitations; perhaps in some of these cases the seat of origin of the rhonchi is too deep for crepitations to be heard from it. Tuberculous rhonchi need to be distinguished from those produced by bronchial catarrh or asthma. The criterion is that they are localized and that the localization is persistent. Bronchitic or asthmatic rhonchi may be localized, but only temporarily. They rapidly disappear from one part of the chest to appear in another; tuberculous rhonchi are constantly detected in the same limited region. It may chance that an advance guard of scattered grey granulations are the offshoot of a focus of infection situared too deeply to afford any direct signs of its existence. In such a case crepitations or clicks, or crepitations with rhonchi, or even rhonchi alone, persistently localized to the apex, may be the only physical evidence of the disease until the multiplication of the granulations is sufficient to impair the percussion note. The importance of not neglecting localized rhonchi is manifest. You will not forget, of course, to make the patient cough before deciding on any question of moist sound. The cough effects two ends: it brings out the moist sound that indicates alveolar exudation and it eliminates moist sounds due merely to bronchial catarrh. When seeking for evidence of tuberculous advance you will, of course, not limit your exploration to the edges or surface of the consolidated patches. The germ may have been carried by the air passages to more distant parts. You will search the entire chest for evidence of fresh granulations, and particularly the special seats of election of the disease.

Even if no moist sounds or rhonchus can be found, it does not altogether follow that the consolidation is in an inactive state. In a small number of cases the lesion is purely bronchopneumonic from the outset; in a further number the more recent advances may be purely broncho-pneumonic; and tuberculous broncho-pneumonia is capable of advancing slowly without giving rise to any moiet sounds at all. The diagnosis between an advancing bronchopneumonic phthisis of smal extent and a quiescent fibrous patch is, therefore, difficult to make by the physical signs alone. Inspection and palpation, and another method of examination that is often neglectedthe measurement of the apices-may assist us here. If over a small patch of consolidation, without any suggestion of a cavity, the chest wall is found to be flattened and the inspiratory movement limited, and if the diminution in girth at the apex as compared with the sound side can be shown by measurement, and if at the same time we find no definite symptoms of active tuberculosis, no rise of temperature, no loss of flesh, no recent history of haemoptysis, for example, the presumption is that it is an old scar and not a recent lesion that is present. But in all such cases the safest plan is to keep the patient under observation for at least a month until you are satisfied that the patch is not extending and that no overt symptoms are about to declare themselves. You will, of course, in such a case make a bacteriological examination of the sputum, if there is any, or of the faucial secretions if there is no expectoration; but, as I have already said, the absence of bacilli is not absolute proof that there is no active lesion, and their presence, unless in large numbers, does not exclude the possibility of the lesion being at the time quiescent and mainly fibrous. Neither physical signs nor bacteriological results, let me warn you in passing, must blind us to the importance of symptoms in cases of doubtful diagnosis. A daily rise of temperature and a progressive loss of body weight, otherwise unaccounted for, are almost formal evidence of the advance of tubercle in a person who has already suffered from the disease, even though the physical signs give no indication of active growth. For reasons I have already entered into, none of our methods of physical examination can completely explore the lung beyond a certain depth, and, owing to accidents of topographical position, extensive advance may be going on without affecting the surface signe in any degree. I do not mean to say that this is common, but it is not so rare that the possibility can be neglected.

The physical signs of large cavities hardly come within the scope of our present subject. Vomicae which produce amphoric hreathing, echoing voice and cough, metallic tinkling and marked pectoriloquy belong to a more advanced period of the tuberculous invasion than that which we are discussing. Small cavities, nevertheless, may exist in cases in which the supericial area of tubercle yielding physical signs is but small. In zome cases of rapid tuberculous invasion cavitation may ensue at an early stage, before the consolidation signs have extended far; in others, a small smooth-walled cavity may exist in an apex which is largely fibrotic and contracted from cicatrized tuberculous lesions. In others yet, a vomica may be a part of a deeply-lying lesion of which only a small area comes near the surface. In all such cases the detection of the cavity is most important, as its existence increases the gravity of the case. It is hardly less important not to mistake the signs of consolidation for those of cavitation, and so to diagnose a vomica where none exists-an error by no means uncommon.

The laws which govern the conduction of the breath and voice sounds indicating consolidation apply equally to those indicating cavities. A cavity covered by a certain depth of healthy or emphysematous lung is dumb; no sign of its pre- 
sence reaches the ear; a cavity connected with the surface by continuous consolidation may, and probably will, give evidence of itself by a peculiar modification of the breath sounds, if not of the voice sounds also, that indicate consolidation. Over cavities of less than a certain size you must not expect to find the true "amphoric" breathing, the sound as of the breath passing over the mouth of a vase, any more than "metallic tinkling " or "echoing cough"; but even over small cavities the breath sound has a definite and readily recognizable peculiarity, which entitles it to the name of "cavernous breathing." Now cavernous breathing is sometimes difficult to make sure of unless you are provided with a definite criterion by which to distinguish it from the tubular breathing of which it is really a modification. Such a criterion is not to be found in the inspiratory part, which differs little, if at all, from that of tubular breathing, but it is furnished by the expiratory part, and the distinction involves a question of pitch. In tubular breathing due to consolidation the expiratory sound is invariably of a slightly lower pitch than the inspiratory; while over a cavity the difference is reversed. Curiously the distinction of pitch does not strike every one alike. The expiratory sound of cavernous, as of tubular, breathing is a "noige" made up of vibrations of very various lengths, and some ears appear to be more sensitive to the higher and some to the lower vibrations among them. The former convey the general impression of a relatively high pitched, the latter that of a relatively low-pitched sound. Some authors, you will accordingly find, represent comparative lowness of pitch, instead of comparative acuteness, as characteristic of the expiratory part of cavernous breathing. I have several times brought the question to a practical test in $\mathrm{my}$ classes in the wards, and every time with the result that to the majority, as to myself, the cavernous expiratory gound appears to have a higher pitch than the inspiratory. Whichever way the difference impresses your individual ear. you will find it constant for nearly all cavities. In most persons, sounds of the character I have described as cavernous may be heard if the stethoscope be applied over the trachea. This suggests a caution in interpreting dry cavernous breathing heard either above the clavicle or over the back near the windpipe. In either case it may chance to be only tracheal breathing conveyed through consolidated lung. Error is best avoided by comparing the supposed cavernous breathing with the actual tracheal sound.

Pectoriloquy is of less value as a sign of cavitation than cavernous breathing. It is not always heard over small cavities, and it is occasionally heard when no cavity exists. I will devote a few words to the definition of this term, as it is not infrequently quite misused. Normal vocal resonance, as I have already said, is a . confused "buzz" conveying a general impression of the vocal sounds uttered, but no distinct idea of the articulation. Vocal resonance may be augmented or may even: become bronchophonic without becoming distinctly articulate. In this case it should not be called pectoriloquy; the term is intended to imply the distinct conveyance of articulate speech to the listener's ear. Pectoriloquial conduction is more easily. detected when the whispered is substituted for the spoken voice, but the mere conduction of the whispered sound, as long as it is in the "buzz" form, does not constitute pectoriloquy, or even fürnish evidence of disease. It often takes place through healthy lung. The sign to which many years ago I gave the name of "post-vocal whiff," I mean the faint short expiratory puff which is sometimes heard when articulation has ceased and the lips of the glottis have flown open to give exit to the compressed air in the trachea, is of no value as a diagnostic mark of a cavity. It sometimes occurs over a cavity, but quite as often over merely consolidated lung in the apex.

Up to this point. I have had young subjects, the most usual victims of tubercle, mainly in mind. But tuberculosis of the lung may break out at any age. In the elderly its first onset has frequently to be recognized by a somewhat different set of signs. After a certain age the disease is more commonly the sequel of a chronic non-tuberculous bronchitis. The bronchitis may have existed on and off for many years, appearing under the guise of a "winter cough" and disappearing in the spring, and the early signs of tubercle have to be recognized among those of a generalized bronchitis which may have become familiar by repetition. The earliest sign to be discerned is usually neither dullness nor harsh breathing, nor alteration of voice sound, but a local change in the character of the bronchitic rhonchus. The pitch of a bronchitic rhonchus in the upper part of the chest, whether inspiratory or expiratory, does not usually vary much throughout the individual inspiration or expiration, whichever it be. If the inspiratory rhonchus at one or other apex become broken in the middle, the latter part becoming markedly high pitched, so as to give to the ear the impression of a creak, it should arrest attention. "Creaking" rhonchus (the creak is more often inspiratory than expiratory) in the adult should always convey a strong suspicion of tuberele. If, in addition, the inspiration should bring out sharp clicks, or even small râles of a higher pitch than is found over the rest of the lung, the proof is almost formal. I am speaking, you will remember, of the adult, and practically of the elderly, subject. In children these signs have no such significance ; they are quite common in ordinary bronchopneumonia. In the elderly I have never, to my recollection, heard them at the apex except in lungs which were subsequently proved to be tuberculous, either by bacteriological examination or by the subsequent progress of the case.

\section{THE EARLY DIAGNOSIS OF TUBERCULOSIS OF THE LUNGS.}

BY C. G. H. BAUMLER, M.D., F.R.C.P.LoND., Professor of Clinical Medicine in the University of Freiburg-i.-Br. formerly Assistant-Physician to the German Hospital, Dalston and the Hospital for Diseases of the Chest, Victoria Park. London.

WITH the progress of our knowledge of pulmonary tuberculosis the desirability or rather the necessity of recognizing in patients the very first changes has greatly increased, more especially since a more hopeful view as regards the curability of the disease has replaced the former despondency. We now know, to a certain extent at least, the way and the means by which the organism gets rid of the changes which the tubercle bacillus brings about, at first quite locally, in the tissues. Local inflammatory reaction and the formation of connective and, further on, of fibrous tissue, which means the replacing the diseased lung-substance by a cicatrix, are the prominent features of this healing process in which, by reactive functions of various kinds, the whole organism is more or less involved. In many cases the original strength of the body is sufficient to fight the battle even amidst unfavourable surroundings, but in the majority of patients in whom already a certain dégree of debility had been the cause that the disease could have taken root, or could have been developed, assistance by external ineans gives the only chance of recovery. Brehmer and Dettweiler, Spengler, Sir Hermann Weber, and other pioneers on the line of the climatic treatment of tuberculosis of the lungs have shown the way by which this external support can best be offered to the patient, and by which unexpected results have already been obtained in ever increasing instances.

But this, no less than other measures-in fact, the whole modern crusade against tuberculosis-can, apart from prophylaxis, only be successful, if patients get under appropriate treatment as early as possible, when only few and small foci of disease are present, and as long as the destruction and replacement of them by fibrous tissue does not materially interfere with the normal aetion of the lungs or unfavourably react on the whole organism.

If by the progress in our knowledge of the disease and of the possibility to battle against it, the power of the phyisician has been greatly extended, his responsibility also has enormously increased, involving, as it does, the paramount duty, if possible, to recognize the very beginnings of the disease. These being at first purely local, and remaining so for various lengths of time, to make out the signs of these local changes will be of special importance.

In the majority of cases a somewhat persisting cough is the only symptom which induces a patient, or more often compels his friends, to seek medical advice. Very commonly, however, other symptoms, subjective as well as objective, are already present, which, in connexion with cough or even without it, may raige a suspicion that tubercles may have formed in the lungs. Foremost among these are febrile symptoms, lassitude, pallor, slight shiverings, night sweats, loss of appetite and weight.

Whenever such symptoms are complained of or suspected in a patient, they have to be carefully investigated, being of great value for the diagnosis, and very often even more still for the opinion which we shall form of the prospects for recovery and of the further progress of the case. Increased attention should be paid to these general symptoms, 